\title{
Decreased Perfusion in the Lateral Wall of the Left Ventricle in PET/CT Studies with ${ }^{13} \mathrm{~N}-$ Ammonia: Evaluation in Healthy Adults
}

\author{
William C. Klingensmith III $^{1}$, Carolyn Noonan², Jack H. Goldberg², Dedra Buchwald ${ }^{3}$, John T. Kimball ${ }^{4}$, \\ and Spero M. Manson ${ }^{5}$ \\ ${ }^{1}$ Hewit Biomolecular Imaging Center, Department of Radiology, Porter Hospital, Denver, Colorado; ${ }^{2}$ Department of Biostatistics, \\ University of Washington, Seattle, Washington; ${ }^{3}$ Department of Medicine, University of Washington, Seattle, Washington; \\ ${ }^{4}$ Cardiovascular Services, Porter Hospital, Denver, Colorado; and ${ }^{5}$ American Indian and Alaska Native Programs, University of \\ Colorado at Denver and Health Sciences Center, Aurora, Colorado
}

This study evaluated the causes of the frequently observed decreased myocardial perfusion in the lateral wall of the left ventricle in PET/CT studies with ${ }^{13} \mathrm{~N}$-ammonia in healthy adults. Methods: Thirty-two adults who were undergoing PET/CT of the heart with ${ }^{13} \mathrm{~N}$-ammonia as part of a study of the effects of posttraumatic stress disorder in American Indians were included. Participants who had evidence of heart disease by a physician-administered questionnaire or by pharmacologic stress during the myocardial perfusion study were excluded. The ratio of counts in the lateral wall relative to the septum at stress and rest were compared with the degree of misalignment between the PET and CT images at the left border of the heart and at the right hemidiaphragm. Results: No participant had evidence of heart disease. For the left heart border, $41 \%$ of participants showed misalignment at rest and $62 \%$ showed misalignment at stress. For the right hemidiaphragm, 28\% showed misalignment at rest and 53\% showed misalignment at stress. Among all participants, the decrease in lateral wall counts relative to the septum correlated with the degree of misalignment of the PET and CT images at the left heart border at rest using both average $(P=0.01)$ and maximum $(P<0.01)$ counts per pixel and at stress using maximum counts per pixel $(P=0.02)$ but not average counts per pixel $(P=0.14)$. There was also a correlation between the degree of misalignment of the PET and CT images at the left heart border and at the right hemidiaphragm at stress $(P=0.01)$ but not at rest $(P=0.09)$. The decreased counts in the lateral wall relative to the septum and the misalignment of the PET and CT images at the left heart border and at the right hemidiaphragm were all greater at stress than at rest (all $P<$ 0.05). In those participants who had no misalignment of the PET and CT images at the left heart border, there was still a residual decrease in relative counts in the lateral wall at both stress and rest, using both average and maximum counts (all $P<$ 0.05). Conclusion: Relative decreased counts in the lateral wall of the heart in PET/CT studies with ${ }^{13} \mathrm{~N}$-ammonia are commonly seen in healthy adults. The decreased counts in the lateral wall appear to be caused by an attenuation artifact from mis-

Received Jan. 10, 2009; revision accepted Oct. 16, 2009.

For correspondence or reprints contact: William C. Klingensmith III, $4720 \mathrm{E}$. Oxford Ave., Englewood, CO 80013.

E-mail: bill.klingensmith@mac.com

COPYRIGHT @ 2009 by the Society of Nuclear Medicine, Inc. alignment at the left border of the heart between the PET and CT images, possibly related to differences in respiratory motion during acquisition of the PET and CT images. In addition, there was a small, but significant, decrease in relative counts in the lateral wall even in participants without misalignment at the left heart border or at the right hemidiaphragm.

Key Words: myocardial perfusion imaging; PET/CT; ${ }^{13} \mathrm{~N}-$ ammonia; lateral wall artifacts; healthy adults

J Nucl Med Technol 2009; 37:215-219

DOI: 10.2967/jnmt.109.062059

$\mathbf{T}$ he development of combined PET/CT scanners has changed the method of generating density maps for attenuation correction of PET images. The previous method of acquiring a density map with an external rotating radioactive source had the disadvantages of requiring considerable time and being of low spatial resolution. On the other hand, the method had the advantage that the blurring effect of respiratory and cardiac motion was the same for the density map as it was for the PET images. The routine CT-acquired density map of the heart is acquired in a much shorter time and results in less blurring. Because the heart moves within the chest with respiration and there is a large difference in density between the heart and adjacent lung, attenuation correction artifacts in the lateral wall of the left ventricle have become common with PET/CT (1-6).

Even with conventional density maps generated with radioactive sources, there are reports of defects in the lateral wall of PET images of myocardial perfusion with ${ }^{13} \mathrm{~N}$-ammonia $(7,8)$. The cause of this finding has never been fully explained, leaving the possibility that the lateral wall defect is a normal finding in PET of the heart with ${ }^{13} \mathrm{~N}$ ammonia (4). In addition, many studies of the lateral wall defect have used patients with known or likely coronary artery disease, making it difficult to separate artifact from disease with certainty $(1,3,8)$. 
The purpose of this study was to evaluate the lateral wall defect in PET/CT myocardial perfusion studies with ${ }^{13} \mathrm{~N}$ ammonia in relation to respiratory motion and other factors in a relatively large number of adults without evidence of coronary artery disease.

\section{MATERIALS AND METHODS}

\section{Participants and Setting}

The research protocol was approved by the Institutional Review Board of the sponsoring institutions. The participants were part of the "Wounded Spirits, Ailing Hearts" study of the effects of posttraumatic stress disorder in American Indians. As part of an extensive evaluation, the participants underwent a physicianadministered assessment that included asking about congenital heart disease, angina, coronary artery bypass surgery, percutaneous coronary angioplasty, and hospitalization for coronary heart disease. A positive answer to any question disqualified a participant from inclusion in this study. In addition, the blood pressure, heart rate, symptoms, and electrocardiograph during pharmacologic stress for PET/CT with ${ }^{13} \mathrm{~N}$-ammonia were reviewed for any findings of coronary artery disease. Any indication of coronary artery disease disqualified the participant.

\section{PET/CT Myocardial Perfusion Imaging}

After a 4-h fast and placement of an intravenous line, the participant was placed in an inline PET/CT scanner with a 4-slice spiral CT component (DLS PET/CT; GE Healthcare). CT images of the lower chest were obtained in approximately $17 \mathrm{~s}$ during quiet breathing followed by the injection of $740 \mathrm{MBq}(20 \mathrm{mCi})$ of ${ }^{13} \mathrm{~N}$-ammonia intravenously. Four and a half minutes later, a 15-min electrocardiograph-gated set of static tomograms was obtained for evaluation of relative myocardial perfusion. The participant was allowed to get up at the end of the rest acquisition. At $45 \mathrm{~min}$, the participant was again placed in the PET/CT scanner and a second CT scan of the lower chest was obtained. The participant was then stressed pharmacologically with a 45- to 60-mg infusion of dipyridamole intravenously over $4 \mathrm{~min}$. Two minutes after the end of the infusion, $740 \mathrm{MBq}(20 \mathrm{mCi})$ of ${ }^{13} \mathrm{~N}$ ammonia were injected. Four and a half minutes later, a 15-min electrocardiograph-gated set of stress tomograms was obtained.

\section{Image Analysis}

The images were analyzed on a Xeleris workstation (GE Healthcare). In the mid portion of the transaxial images of the left ventricle, a vertically oriented rectangular region of interest was manually placed over the lateral wall and the average and maximum counts per pixel were recorded. The region of interest was then translated horizontally to overlie the septum, and again the average and maximum counts per pixel were recorded.

Then, using the original PET, CT, and fused images obtained transaxial to the body, the maximum extent to which the left ventricle in the PET image extended into the lung in the corresponding CT image was measured. In addition, the number of slices between the top of the right hemidiaphragm in the PET images and CT images was recorded as an indication of misalignment in the cephalocaudad direction.

\section{Statistical Analysis}

We assessed the correlation among the ratio of lateral wall counts relative to septal counts, the amount of misalignment at the left heart border, and the amount of misalignment at the top of the right hemidiaphragm using the Pearson correlation coefficient. Descriptive statistics for continuous variables included mean, SD, and range; percentages were computed for categoric variables.

Correlations were computed among counts in the lateral wall of the heart relative to the septum, degree of misalignment measured at the lateral heart border, and degree of misalignment at the top of the right hemidiaphragm while participants were at rest and under pharmacologic stress during imaging. A negative correlation indicates that as misalignment increases, the counts in the lateral wall relative to the septum decrease. For completeness, we present results for both average and maximum counts despite a strong correlation between the measures $(r=0.69$ at rest, $r=0.74$ at stress).

Paired $t$ tests were used to compare the ratio of lateral wall counts relative to septal counts at rest and at stress. We also used paired $t$ tests to compare the amount of misalignment at rest and at stress. In participants without misalignment at the left heart border, we used 1-sample $t$ tests to examine if the ratio of lateral wall counts relative to septal counts differed from 1.0. A significant result indicates decreased counts in the lateral wall relative to the septum.

Because measures for the amount of misalignment did not follow a normal distribution, we also computed nonparametric equivalents to Pearson correlation (Spearman rank) and the paired $t$ test (Wilcoxon signed ranks). For consistency, we present results from the parametric tests because statistical inference did not differ between the parametric and nonparametric tests.

Analyses were completed using Stata/SE 9.2 (StataCorp LP) for Windows (Microsoft). All statistical comparisons were 2-sided, and we used a $P$ value of 0.05 as the threshold for significance.

\section{RESULTS}

\section{Participants}

All 32 participants had no history or current evidence of heart disease and were included in analyses. Most of the participants were female $(81 \%)$, and the average age was $44 \pm 9$ y (range, 28-61 y).

\section{Data Analysis}

The amount of misalignment at the left heart border ranged from 0.0 to $11.3 \mathrm{~mm}$ at rest and 0.0 to $18.0 \mathrm{~mm}$ at stress. For the left heart border, $41 \%$ of participants at rest and $62 \%$ of participants at stress had misalignment $(>0$ $\mathrm{mm}$ ). The amount of misalignment at the top of the right hemidiaphragm ranged from 0 to 2 slices at rest and 0 to 4 slices at stress. For the top of the right hemidiaphragm, $28 \%$ of participants at rest and $53 \%$ of participants at stress had misalignment ( $>0$ slices).

Correlations among the ratio of lateral wall counts relative to septal counts, misalignment at the left heart border, and misalignment at the top of the right hemidiaphragm are shown in Table 1. At rest, we found significant negative correlations between lateral wall counts relative to the septum and misalignment at the left heart border for both average $(r=-0.45)$ and maximum $(r=-0.52)$ counts. The same association at stress was significant for maximum $(r=-0.43)$, but not average ( $r=-0.26)$, counts. The correlation at rest between the amount of misalignment at the left heart border and the 
TABLE 1

Correlations Among Relative Lateral Wall Counts and Misalignments at Left Heart Border and Top of Right Hemidiaphragm

\begin{tabular}{|c|c|c|}
\hline Comparison & $r$ & $P$ \\
\hline \multicolumn{3}{|c|}{ Counts in lateral wall relative to septum vs. misalignment at left heart border } \\
\hline \multicolumn{3}{|l|}{ Rest } \\
\hline Using average counts per pixel within region of interest & -0.45 & 0.01 \\
\hline Using maximum counts per pixel within region of interest & -0.52 & $<0.01$ \\
\hline \multicolumn{3}{|l|}{ Stress } \\
\hline Using average counts per pixel within region of interest & -0.26 & 0.14 \\
\hline Using maximum counts per pixel within region of interest & -0.43 & 0.02 \\
\hline \multicolumn{3}{|c|}{ Misalignment at left heart border vs. misalignment at top of right hemidiaphragm } \\
\hline Rest & 0.31 & 0.09 \\
\hline Stress & 0.45 & 0.01 \\
\hline \multicolumn{3}{|c|}{ Counts in lateral wall relative to septum vs. misalignment at top of right hemidiaphragm } \\
\hline \multicolumn{3}{|c|}{ Rest } \\
\hline Using average counts per pixel within region of interest & -0.14 & 0.45 \\
\hline Using maximum counts per pixel within region of interest & -0.04 & 0.84 \\
\hline \multicolumn{3}{|l|}{ Stress } \\
\hline Using average counts per pixel within region of interest & -0.09 & 0.63 \\
\hline Using maximum counts per pixel within region of interest & -0.13 & 0.49 \\
\hline
\end{tabular}

amount of misalignment at the top of the right hemidiaphragm was positive $(r=0.31)$ but not significant $(P=0.09)$. However, the same correlation at stress was significant ( $r=0.45, P=0.01)$.

Correlations between the lateral wall counts relative to the septum and the amount of misalignment at the top of the right hemidiaphragm were smaller than analogous associations at the left heart border and not significant.

Differences between rest and stress in the relative counts in the lateral wall, compared with the septum, the degree of misalignment at the left heart border, and the degree of misalignment at the top of the right hemidiaphragm, are presented in Table 2. Participants had a significantly lower lateral wall count relative to the septum at stress than at rest for both average $(P<0.01)$ and maximum $(P=0.02)$ counts, greater misalignment at the left heart border at stress than at rest $(P=0.02)$, and greater misalignment at the top of the right hemidiaphragm at stress than at rest $(P<0.01)$.

Finally, we examined the counts in the lateral wall relative to the septum in participants with no misalignment at the left heart border (Table 3). Among the 19 participants with no misalignment at rest, the average and maximum counts were lower in the lateral wall than in the septum ( $P=0.03$ and $P=0.01$, respectively). Among the 12 participants with no misalignment at stress, both the average and the maximum counts were lower in the lateral wall than in the septum $(P<0.01$ for both).

\section{DISCUSSION}

This study, based on 32 adults without evidence of heart disease, demonstrates that the relative decreased counts in the lateral wall of the left ventricle of the heart seen in PET/ CT studies with ${ }^{13} \mathrm{~N}$-ammonia correlates with misalignment of the PET and CT images at the left heart border. This misalignment appears to be related to differences in respiratory motion during acquisition of the PET and CT images. Furthermore, the decreased counts in the lateral wall relative to the septum, and misalignment of the left heart border and top of the right hemidiaphragm, were all worse at stress. Of note, in participants without evidence of misalignment at the left heart border, the counts in the lateral wall were less than in the septum and this disparity increased at stress.

Relatively decreased counts in the lateral wall in PET perfusion studies with ${ }^{13} \mathrm{~N}$-ammonia were observed even before the development of combined PET/CT scanners (8). However, this phenomenon appears to be more common with PET/CT scanners (1-8). In our patients, $41 \%$ showed misalignment at the left heart border at rest and $62 \%$

TABLE 2

Comparison of Relative Lateral Wall Counts and Misalignments at Stress and at Rest

\begin{tabular}{|c|c|c|c|}
\hline Parameter & Stress & Rest & $P$ \\
\hline Lateral wall relative to septum using average counts & $0.79(0.11)$ & $0.86(0.16)$ & $<0.01$ \\
\hline Lateral wall relative to septum using maximum counts & $0.87(0.11)$ & $0.92(0.09)$ & 0.02 \\
\hline Misalignment at left heart border (mm) & $4.23(5.21)$ & $1.85(3.14)$ & 0.02 \\
\hline Misalignment at top of right hemidiaphragm (slices) & $1.03(1.18)$ & $0.31(0.54)$ & $<0.01$ \\
\hline
\end{tabular}


TABLE 3

Counts in Lateral Wall Relative to Septum in Patients Without Left Heart Border Misalignment at Stress and at Rest

\begin{tabular}{|c|c|c|c|}
\hline Parameter & Mean & Decrease $^{*}$ & $P$ \\
\hline \multicolumn{4}{|l|}{ Rest $(n=19)$} \\
\hline Lateral wall relative to septum using average counts & $0.90(0.18)$ & $10 \%$ & 0.03 \\
\hline Lateral wall relative to septum using maximum counts & $0.95(0.07)$ & $5 \%$ & 0.01 \\
\hline \multicolumn{4}{|l|}{ Stress $(n=12)$} \\
\hline Lateral wall relative to septum using average counts & $0.79(0.09)$ & $21 \%$ & $<0.01$ \\
\hline Lateral wall relative to septum using maximum count & $0.91(0.09)$ & $9 \%$ & $<0.01$ \\
\hline
\end{tabular}

SD is in parentheses.

${ }^{*}$ Percentage decrease in counts in lateral wall relative to septum.

showed misalignment at the left heart border at stress. In addition, 28\% showed misalignment at the top of the right hemidiaphragm at rest, whereas 53\% showed misalignment at the top of the right hemidiaphragm at stress. A significantly greater percentage of participants had misalignment at the top of the right hemidiaphragm at stress than at rest, most likely resulting from an increase in respiratory motion from the effects of pharmacologic stress.

The correlation between the relative decreased counts in the lateral wall of the left ventricle and the degree of misalignment between the PET and CT images at the lateral border of the heart, as well as the correlation between misalignment at the left heart border and at the top of the right hemidiaphragm, suggests that the decreased counts in the lateral wall of the left ventricle are secondary to an attenuation artifact that, in turn, may be secondary to respiratory motion.

Several maneuvers have been suggested to prevent or correct misalignment of the PET and CT images with respect to the heart, such as increasing the time of acquisition of the CT images over multiple respiratory cycles to create the same respiratory averaging present in the PET images (9-11). Others have suggested acquiring the CT images at end expiration, when the lateral border of the heart would be expected to extend to the left to the greatest extent (12). For stress images, some have tried respiratory gating or acquiring the $\mathrm{CT}$ image after the PET acquisition so that the effect of pharmacologic stress on breathing will have decreased (13-16). The most reliable approach, however, is probably the use of software to manually coregister the PET and CT images or to manually adjust all CT pixels that correspond to the heart in the PET images to the density of the heart in the CT images $(2,8,17-19)$.

It has been suggested that the decreased perfusion in the lateral wall is a physiologic finding that is perhaps due to a local difference in the metabolism of ammonia (6). Others have not found a decrease in perfusion in the lateral wall after optimal attenuation correction (1). Our data indicate a small, but significant, decrease in perfusion in the lateral wall relative to the septum even in participants without any discernible misalignment of the PET and CT images at the lateral border of the heart. This finding may be physiologic or due to factors other than detectable misalignment.

A noteworthy limitation of this study was the lack of robust software options in the Xeleris workstation for creating regions of interest and measuring counts in any desired portion of the left ventricle. This limitation may account for some of the statistically insignificant findings between parameters that would be expected to be strongly associated, such as relatively decreased counts in the lateral wall versus misalignment of the top of the right hemidiaphragm.

PET/CT has substantial advantages over conventional nuclear medicine imaging with $\gamma$-cameras. It has higher and more uniform spatial resolution, better CT correlation for anatomic localization, and, because of the greater variety of potential radiopharmaceuticals, the ability to image metabolism as well as physiology. However, despite the desirability of moving nuclear medicine myocardial imaging to PET/CT to take advantage of its superior technology, the frequent lateral wall artifact with current equipment and protocols is a limiting factor. We hope that manufacturers will develop software applications that will allow easy correction of misalignment between PET and CT images.

\section{CONCLUSION}

This study provided evidence that decreased counts in the lateral wall of the left ventricle in PET/CT myocardial studies with ${ }^{13} \mathrm{~N}$-ammonia occur frequently and are related to an attenuation artifact caused by misalignment of the PET and CT images at the left heart border. The misalignment at the left heart border seems to be related to respiratory motion and is more marked at stress. Finally, in those participants without evidence of misalignment at the left heart border, we observed a residual small decrease in lateral wall counts, compared with the septum.

\section{ACKNOWLEDGMENTS}

Financial support was provided by "Wounded Spirits, Ailing Hearts: Post-Traumatic Stress Disorder and Cardiovascular Disease in Indians," a trial sponsored by the National Heart, Lung, and Blood Institute (RO1 HL073824). 


\section{REFERENCES}

1. Koepfli P, Hany TF, Wyss CA, et al. CT attenuation correction for myocardial perfusion quantification using a PET/CT hybrid scanner. J Nucl Med. 2004;45:537-542.

2. Schwaiger M, Ziegler S, Nekolla SG. PET/CT: challenge for nuclear cardiology. J Nucl Med. 2005;46:1664-1678.

3. Martinez-Moller A, Souvatzoglou M, Navab N, et al. Artifacts from misaligned CT in cardiac perfusion PET/CT studies: frequency, effects, and potential solutions. J Nucl Med. 2007;48:188-193.

4. Cook RAH, Carnes G, Lee TY, et al. Respiration-averaged CT for attenuation correction in canine PET/CT. J Nucl Med. 2007;48:811-818.

5. Alessio AM, Kohlmyer S, Branch K, et al. Cine CT for attenuation correction in cardiac PET/CT. J Nucl Med. 2007;48:794-801.

6. Di Carli MF, Dorbala S, Meserve J, et al. Clinical myocardial perfusion PET/CT. J Nucl Med. 2007;48:783-793.

7. de Jong RM, Blanksma PK, Willemsen ATM, et al. Posterolateral defect of the normal human heart investigated with nitrogen-13-ammonia and dynamic PET. J Nucl Med. 1995;36:581-585.

8. Loghin C, Sdringola S, Gould KL. Common artifacts in PET myocardial perfusion images due to attenuation-emission misregistration: clinical significance, causes, and solutions. J Nucl Med. 2004;45:1029-1039.

9. Pan T, Mawlawi O, Nehmeh SA, et al. Attenuation correction of PET images with respiration-averaged CT images in PET/CT. J Nucl Med. 2005;46:1481-1487.

10. Brunken RC, DiFilippo FP, Bybel B, et al. Clinical evaluation of cardiac PET attenuation correction using "fast" and "slow" CT images [abstract]. J Nucl Med. 2004;45(suppl):120P.
11. Nye JA, Votaw JR, Esteves F. Attenuation correction of cardiac PET/CT with gated, fast, and slow acquisition of the transmission map [abstract]. J Nucl Med. 2006;47(suppl):132P.

12. Bacharach SL. PET/CT attenuation correction: breathing lessons. J Nucl Med. 2007;48:677-679.

13. Dorbala S, Limaye A, Sampson U, et al. Optimal timing of transmission MAP for rubidium 82 stress positron emission tomography (PET-CT) myocardial perfusion imaging [abstract]. $J$ Nucl Med. 2005;46(suppl):267P.

14. Youssef IM, Colon D, Rafique A, et al. The benefit of two CT transmission scans for rubidium-82 cardiac PET myocardial perfusion imaging [abstract]. $\mathrm{J} \mathrm{Nucl}$ Med. 2005;46(suppl):115P.

15. Livieratos L, Rajappan K, Stegger L, et al. Respiratory gating of cardiac PET data in list-mode acquisition. Eur J Nucl Med Mol Imaging. 2006;33:584588.

16. Lautamäki R, Brown TL, Merrill J, Bengel FM. CT-based attenuation correction in ${ }^{82} \mathrm{Rb}$-myocardial perfusion PET-CT: incidence of misalignment and effect on regional tracer distribution. Eur J Nucl Med Mol Imaging. 2008;35:305-310.

17. Gould KL, Pan T, Loghin C, et al. Frequent diagnostic errors in cardiac PET/CT due to misregistration of CT attenuation and emission PET images: a definitive analysis of causes, consequences, and corrections. J Nucl Med. 2007;48:11121121.

18. Wilson JW, Wong TZ, Borges-Neto S, et al. An algorithm for correction of PET/ CT mismatch induced cardiac attenuation correction artifacts [abstract]. $\mathrm{J} \mathrm{Nucl}$ Med. 2005;46(suppl):55P.

19. Nehmeh SA, Yusuf EE. Respiratory motion in positron emission tomography/ computed tomography: a review. Semin Nucl Med. 2008;38:167-176. 